\title{
Otohematoma canino: Análise Retrospectiva de Ocorrências Atendidas no Hospital Veterinário das Faculdades Integradas Aparício Carvalho - FIMCA
}

Canime Otohematoma: Retrospective Analysis of Occurrences Attended at the Veterinary Hospital of Faculdades Integradas Aparício Carvalho - FIMCA

Otohematoma canino: análisis retrospectivo de ocurrencias atendidas en el Hospital Veterinario de las Faculdades Integradas Aparício Carvalho - FIMCA

Recebido: 18/06/2021 | Revisado: 23/06/2021 | Aceito: 08/07/2021 | Publicado: 18/07/2021

\author{
Sabrina Benigna Almeida \\ ORCID: https://orcid.org/0000-0002-5365-5866 \\ Centro Universitário Aparício Carvalho, Brasil \\ E-mail: sabrineixonsbenigna@gmail.com \\ Matheus de Almeida Rocha \\ ORCID: https://orcid.org/0000-0002-2278-7532 \\ Centro Universitário Aparício Carvalho, Brasil \\ E-mail: matheusalmeidarocha23@gmail.com \\ Thiago Vaz Lopes \\ ORCID: https://orcid.org/0000-0002-3192-1908 \\ Universidade Federal do Acre, Brasil \\ E-mail: thiagovlopes@ hotmail.com \\ João Gustavo da Silva Garcia de Souza \\ ORCID: https://orcid.org/0000-0002-5899-2490 \\ Centro Universitário Aparício Carvalho, Brasil \\ E-mail: joaogarciadesouza27@gmail.com \\ Sandro de Vargas Schons \\ ORCID: https://orcid.org/0000-0001-9811-5356 \\ Universidade Federal de Rondônia, Brasil \\ E-mail: sandroschons@unir.br
}

\begin{abstract}
Resumo
O otohematoma canino, também chamado de hematoma aural ou auricular, é uma das patologias mais frequentes nos atendimentos clínicos, sendo a lesão que mais atinge o aparelho auditivo dos cães. Descreve-se pelo acúmulo de líquido seroso e sangue entre a cartilagem e a epiderme do ouvido externo causando desconforto nos animais. Este trabalho teve como objetivo analisar a epidemiologia do hematoma auricular em cães atendidos e registrados em fichas no Hospital Veterinário das Faculdades Integradas Aparício Carvalho - FIMCA, na cidade de Porto Velho, Rondônia no período de Janeiro de 2017 a Novembro de 2018. Esse estudo dos dados foi alcançado por meio das fichas cadastradas de todos os pacientes atendidos e diagnosticados por otohematomas, utilizando fatores que podem predispor a presença desta afecção, como raça, sexo ou porte físico. Foram diagnosticados 13 cães com a patologia, comprovando 2 picos de maior ocorrência, equivalentes aos meses de Setembro (2 casos;15,3\%) e Dezembro (2 casos;15,3\%) de 2017. Animais sem raça definida obtiveram maior número de afecções $(9 / 13 ; 69,2 \%)$ e cães do gênero masculino prevaleceram $(53,8 \% ; 7 / 13)$. A maioria dos animais apresentavam otohematoma bilateral $(11 / 13 ; 84,6 \%)$. Em vista disso, na rotina clínica do hospital veterinário analisado o otohematoma canino apresentou relevante número de ocorrências, sendo mais comum em animais sem raça definida e machos, na qual esta enfermidade demonstrou ser um fator de risco em animais de médio e grande porte. O estudo permitiu demonstrar maior predomínio de hematoma auricular bilateral e as maiores prevalências de casos nos meses de Setembro e Dezembro de 2017.
\end{abstract}

Palavras-chave: Otohematoma; Cães; Epidemiologia; Orelha; Prevalência.

\begin{abstract}
Canine otohematoma, also called aural or auricular hematoma, is one of the most frequent pathologies in clinical care, being the most affected lesion in dogs. It is described by the accumulation of serous liquid and blood between the cartilage and the epidermis of the external ear causing discomfort in the animals. The objective of this study was to analyze the epidemiology of atrial hematoma in dogs seen and recorded in the Hospital Veterinário das Faculdades Integradas Aparício Carvalho - FIMCA, in the city of Porto Velho, Rondônia, in the period from January 2017 to November 2018. data were obtained through the registries of all patients seen and diagnosed by otohematomas, using factors that may predispose the presence of this affection, such as race, gender or physical size. A total of 13 dogs were diagnosed with the disease, showing two peaks with the highest occurrence, equivalent to the months of September ( 2 cases, $15.3 \%)$ and December ( 2 cases, $15.3 \%)$ of 2017. Undefined animals obtained higher numbers of affections (9/13,
\end{abstract}


$69.2 \%)$ and male dogs prevailed $(53.8 \%, 7 / 13)$. Most of the animals presented bilateral otohematoma $(11 / 13 ; 84.6 \%)$. Therefore, in the clinical routine of the veterinary hospital analyzed the canine otohematoma presented a relevant number of occurrences, being more common in animals with no defined breed and males, in which this disease was shown to be a risk factor in medium and large animals. The study showed a greater prevalence of bilateral atrial hematoma and the higher prevalence of cases in the months of September and December 2017.

Keywords: Otohematoma; Dogs; Epidemiology; Ear; Prevalence.

\section{Resumen}

El otohematoma canino, también llamado hematoma aural o auricular, es una de las patologías más frecuentes en la atención clínica, siendo la lesión que más afecta al sistema auditivo de los perros. Se describe por la acumulación de líquido seroso y sangre entre el cartílago y la epidermis del oído externo, provocando malestar en los animales. Este estudio tuvo como objetivo analizar la epidemiología del hematoma auricular en perros tratados y registrados en los registros del Hospital Veterinario de las Faculdades Integradas Aparício Carvalho - FIMCA, en la ciudad de Porto Velho, Rondônia, de enero de 2017 a noviembre de 2018. Los datos fueron Fichas registradas de todos los pacientes atendidos y diagnosticados de otohematomas, utilizando factores que pueden predisponer a la presencia de esta afección, como la raza, el sexo o la talla física. Trece perros fueron diagnosticados con la patología, comprobándose 2 picos de mayor ocurrencia, equivalentes a los meses de septiembre (2 casos; $15,3 \%)$ y diciembre (2 casos; $15,3 \%)$ de 2017 . Los animales de raza mixta presentaron mayor número de afecciones. $(9 / 13 ; 69,2 \%)$ y prevalecieron los machos $(53,8 \%$; 7/13). La mayoría de los animales tenían otohematoma bilateral (11/13; 84,6\%). Ante esto, en la rutina clínica del hospital veterinario analizado, el otohematoma canino presentó un número relevante de ocurrencias, siendo más común en animales mestizos y machos, en los que esta enfermedad demostró ser un factor de riesgo en animales medianos y grandes. El estudio permitió demostrar un mayor predominio de hematoma auricular bilateral y la mayor prevalencia de casos en los meses de septiembre y diciembre de 2017.

Palabras clave: Otohematoma; Perros; Epidemiología; Oído; Predominio.

\section{Introdução}

O aparelho auditivo tem como função apanhar, evidenciar e identificar os sons do ambiente (Fossum et al., 2007) e, simultaneamente junto ao restante do corpo funcionar como artifício de exteriorização e comunicação entre os animais (Aspinall et al., 2004). As pesquisas epidemiológicas para patologias em cães indicam que as enfermidades dos ouvidos variam entre 7,5 e 16,5\% na rotina das clínicas veterinárias (Harvey et al., 2005; Rodrigues, et al. 2019), desta forma o otohematoma canino, também chamado hematoma aural ou auricular, é uma das patologias mais frequentes nestes atendimentos, sendo a lesão que mais atinge o aparelho auditivo destes animais (Lanz \& Wood, 2004). Descreve-se pelo acúmulo de líquido seroso e sangue entre a cartilagem e a epiderme do ouvido externo causando desconforto, tendo como etiologia que suscinta em cães: ectoparasitas, traumas, corpos estranhos, crescimento de tecido anormal e inflamações no canal auditivo (Cechner, 2005; Santos \& Guimarães, 2020; Guillemo et al. 2020). Assim como as sarnas auriculares, eczemas atópicos, alergias, aumento da sensibilidade do pavilhão auditivo (Griffin, 1994) e movimentação de modo súbito da cabeça equivalente ao prurido e dor nas orelhas (McCarthy \& McCarthy, 1994; Cechner, 2005; Blattler et al., 2007).

De acordo com o porte físico do cão, do quadro da doença e sua condição em consequência do traumatismo que originou (Kuwahara, 1986; Cechner, 2005; Joyce, 2000) estes hematomas aurais apresentam aparência patognomónica (Harvey et al., 2005), podendo manifestar-se nas formas de massas com crescimentos anormais e proporções diversas, lesões flutuantes, doloridas e edemaciadas (Wilson, 1983; Griffin, 1994). Seu diagnóstico é fundado no histórico dos canídeos atendidos e principalmente nos exames físicos (Griffin, 1994; Joyce, 2000; Cechner, 2005).

Alguns métodos podem ser utilizados como forma de tratamento para o otohematoma. Dentre eles, pode-se citar a técnica de drenagem com agulha como a mais simples e para casos leves (Lanz \& Wood, 2004), incisão drenagem e suturas para os casos onde se observa uma cronicidade do quadro (Krahwinkel, 2003) e a utilização de drenagem e colocação de drenos, sendo utilizados para obter uma drenagem por um tempo maior, com a possibilidade de uso somente com a fibrina no hematoma estando no mínimo (Lanz \& Wood, 2004). Com o passar dos anos foram surgindo diferentes técnicas para o tratamento do 
hematoma auricular, como casos de tratamentos com homeopáticos (Valle, Kanayama \& Nader, 2015) e tratamentos à base de corticóides intralesional (Pachaly, et al. 2021).

Comumente encontrado dentro da rotina da clínica de pequenos animais, o otohematoma e as demais patologias do pavilhão auricular canino se mostram cada vez mais registradas em clínicas e hospitais veterinários. Sendo assim, é de extremamente relevância a compilação e análise desses dados, para que se tenha uma percepção cada vez mais aprimorada sobre a relevância do hematoma auricular dentro da rotina veterinária de pequenos animais (Evangelista et al. 2012).

Este trabalho teve como objetivo registrar a epidemiologia do hematoma auricular em cães atendidos e registrados em fichas no Hospital Veterinário das Faculdades Integradas Aparício Carvalho - FIMCA, na cidade de Porto Velho, Rondônia, utilizando fatores que podem predispor a presença desta afecção, como raça, sexo ou porte físico.

\section{Materiais e Métodos}

Esta análise foi desempenhada no Hospital Veterinário das Faculdades Integradas Aparício Carvalho - FIMCA, no munícipio de Porto Velho - RO, durante o decorrer de janeiro de 2017 a agosto de 2018, sendo uma pesquisa de natureza quantitativa sobre o hematoma auricular. A análise dos dados foi alcançada por meio de registros das fichas cadastradas de todos os pacientes atendidos, onde foram acompanhados todos os cães diagnosticados por otohematoma e encaminhados ao tratamento clínico pelos médicos veterinários. Foram obtidas informações sobre a epidemiologia desta afecção como: raças regularmente acometidas, meses de maior ocorrência, sexo (macho ou fêmea), porte físico e se houve comprometimento de uma ou ambas as orelhas (unilateral ou bilateral). Constatando-se os resultados através de meio estatístico com base em metodologias descritivas com o auxílio das tabelas de frequências e percentuais, obtidas através das informações presentes nos prontuários estudados nos critérios analisados.

\section{Resultados e Discussão}

Ao longo dos anos de 2017 a 2018, foram examinados inúmeros cães no Hospital Veterinário - FIMCA, dos quais 13 cães foram diagnosticados com otohematoma, onde foram registrados dados epidemiológicos durante o período de Janeiro de 2017 a Novembro de 2018 (totalizando 23 meses) para levantamento de dados. Em relação a prevalência de casos registrados, verificou-se que os números ao longo dos anos diversificaram, comprovando 2 picos de maior ocorrência, que equivaleram aos meses Setembro ( 2 casos) e Dezembro ( 2 casos) de 2017, com percentagem de 15,3\% em comparação ao total (Figura 1). 
Figura 1 - Relacionado à distribuição temporal dos casos diagnosticados por otohematomas no Hospital Veterinário das Faculdades Integradas Aparício Carvalho - FIMCA, ao longo dos anos de 2017 a 2018 (n=13).

\section{Número de casos diagnosticados por otohematoma nos anos de 2017 e 2018}

\section{2,5}

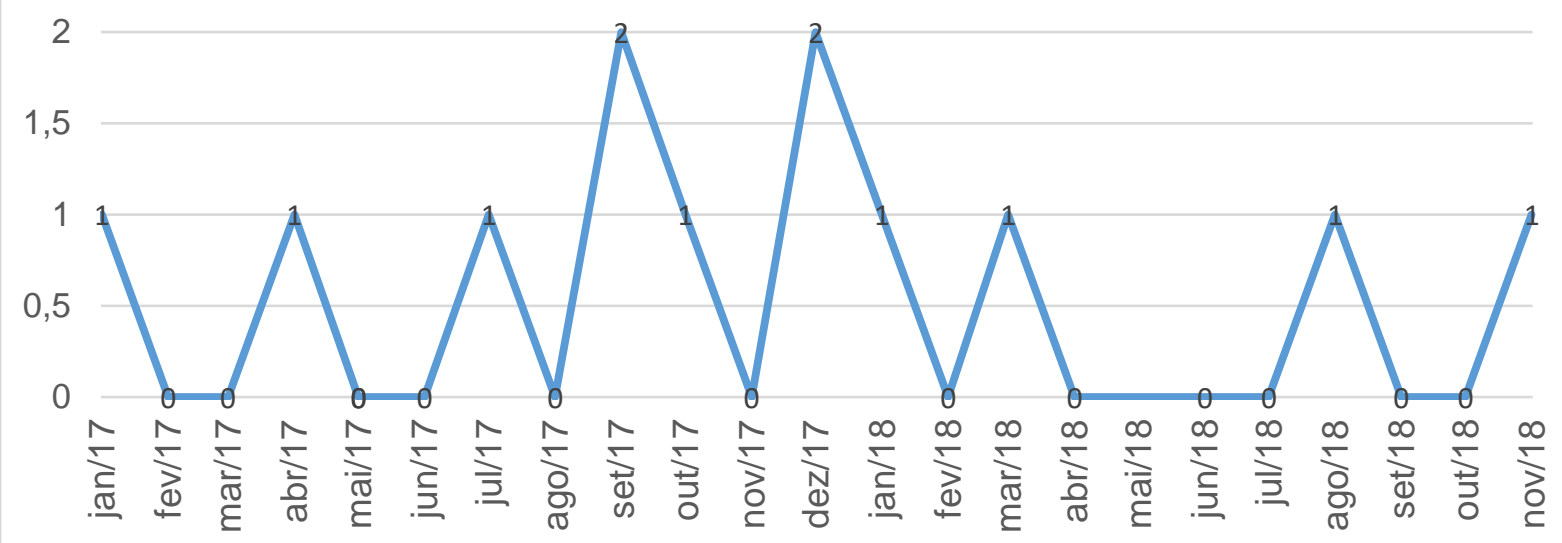

_ Número de casos diagnosticados por otohematoma nos anos de 2017 e 2018

Fonte: Elaborado conforme os resultados de dados coletados nessa pesquisa.

Quanto ao critério de raças mais acometidas, constata-se que os valores variaram entre os anos observados, sendo o padrão racial não definido (Sem Raça Definida) obteve maior número de afecções, com 9 casos diagnosticados (69,2\%). Bem como a raça Pitbull registrou 2 ocorrências (15,3\%), seguindo-se das raças Fila Brasileiro e Labrador Retriever, fichando apenas 1 caso cada (7,6\%) (Figura 2). Evidenciou-se o fato dos cães sem raça definida serem os mais acometidos por serem os maiores números de casos atendidos na prática habitual do Hospital Veterinário - FIMCA, tendo como demais estudos demonstrando igualmente que estes animais foram os mais afetados pelo otohematoma canino (Santos, 2008; Teixeira et al. 2002), supostamente em consequência do aparecimento frequente de otite de secreção ceruminosa (Yoshida et al., 2002). Entretanto, Mikawa et al. (2005) e Joyce (2000) indicaram Labradores Retriever como exemplo das raças mais propensos a serem atingidas.Desta maneira nota-se nos atendimentos do Hospital Veterinário-HVET ser habitual casos de acometimento pela patologia em cães de médio e grande, tal como em outros trabalhos observados (Teixeira et al., 2002; Mikawa et al., 2005; Santos, 2008). 
Figura 2 - Dados epidemiológicos referentes aos padrões raciais de cães acometidos por otohematoma no Hospital Veterinário das Faculdades Integradas Aparício Carvalho durante o período de 2017 a 2018 (n= 13).

\section{Raças caninas acometidas por otohematoma}

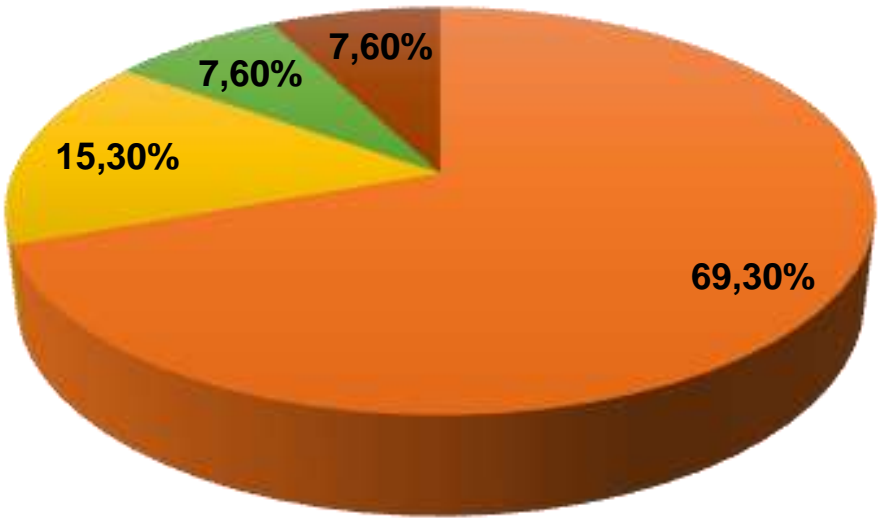

- Sem raça definida $\backsim$ Pitbull $\quad$ Fila Brasileiro $\quad$ Labrador Retriever

Fonte: Elaborado conforme os resultados de dados coletados nessa pesquisa.

Em relação ao sexo dos animais mais atingidos pela enfermidade, obteve-se maior porcentagem em machos (53,8\%;7/13), quando comparadas a fêmeas diagnosticadas (46,1\%; 6/13) (Fig.3). Segundo os estudos de Moya (2004), não é plausível comprovar a causa da maior prevalência do otohematoma canino no gênero masculino, mas suscitou-se que estes se apresentam mais expostos a possíveis traumas físicos devido aos confrontos para acasalamento e territoriais. Ao mesmo tempo, demais análises sustentam que assim como os machos são afetados, quanto as fêmeas, não ocorrendo tendência para exposição quanto ao sexo (Joyce, 2000), sendo possivelmente apenas um fato simplesmente eventual (Santos, 2008).

Figura 3 - Quanto ao gênero sexual mais acometido por otohematoma durante o período de levantamento de dados analisados no Hospital Veterinário das Faculdades Integradas Aparício Carvalho (n=13).

\section{Gênero sexual}

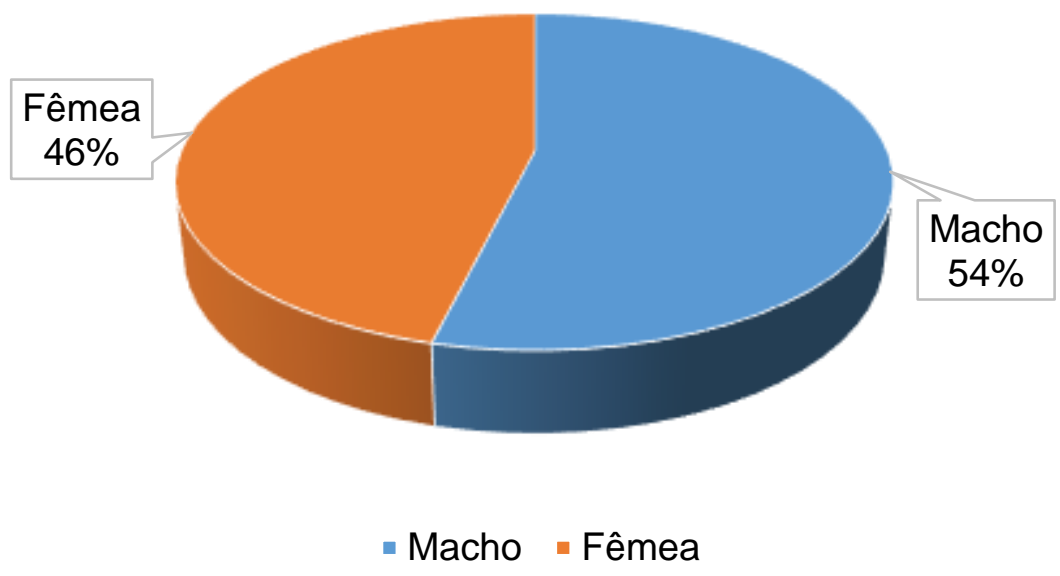

Fonte: Elaborado conforme os resultados de dados coletados nessa pesquisa. 
Correlação a apenas uma pina ou ambas terem sido acometidas por este hematoma aural, observou-se que a maioria dos animais desta pesquisa tiveram lesões tanto na orelha direita, quanto a esquerda (bilateral) com 11 casos (84,6\%). Apenas 15,3\% (2/13) apresentaram otohematoma unilateral.

Os resultados alcançados no atual estudo sugerem que o hematoma auricular canino possui alguns possíveis fatores que predispõe risco a traumas auriculares, como raça e porte físico do animal. Outros estudos analíticos que permitissem acompanhar casos desta patologia em todos os estágios seriam de grande facilidade para investigação, desde sua etiologia à correção terapêutica desta enfermidade.

\section{Considerações Finais}

Em vista disso, na rotina clínica do hospital veterinário analisado o otohematoma canino apresentou relevante número de ocorrências, sendo mais comum em animais sem raça definida e machos, na qual esta enfermidade demonstrou ser um fator de risco em animais de médio e grande porte. O estudo permitiu demonstrar maior predomínio de hematoma auricular bilateral e as maiores prevalências de casos nos meses de Setembro e Dezembro de 2017.

Com base nos dados coletados e nos autores ao qual a pesquisa usou como base, vê-se de uma importância a continuidade de trabalhos referentes a otohematomas, por ser algo comumente encontrado dentro da área de clínica de pequenos animais, utilizando levantamento de dados e a busca por novas alternativas para o tratamento.

\section{Referências}

Aspinall, V., O’reilly, M. \& Capello, M. (2004). Introduction to Veterinary Anatomy and Physiology. Butterworth - Heinenamann.

Blättler U. et al. (2007). Fibrin sealant as a treatment for canine aural haematoma: a case history. Journal Veterinary, 173(3): 697- 700.

Cechner, P. E. (2005). Técnica de sutura para reparo de um otohematoma. in: técnicas atuais em cirurgia de eurides d et al. rpcv Pequenos Animais, (3a ed.i), Roca.

Evangelista, L. S. M. et al. (2012). Estudo retrospectivo do otohematoma em cães atendidos em um hospital veterinário Universitário. Acta Veterinaria Brasilica, $6(1): 48-51$

Fossum, T. et al. (2007). Small Animal Surgery (3a ed.).: Mosby Elsevier.

Griffin, C. (1994). Pinnal Diseases. Veterinary clinics of north américa: Small Animal Practice, 24(5): 897-904.

Guillemo, B. et al. (2020). Pseudomonas aeruginosa em la otitis externa canina: situación actual. ANALECTA VETERINARIA, 40(1).

Harvey, R. G., Harari, J. \& Delauche, A. J. (2005). Ear diseases of the dog and cat. Manson Publishing.

Joyce, J. (2000). Canine aural haematoma. Waltham focus, 10: 4-9.

Krahwinkel, D.J. (2003). Ear. In D., Slatter (Ed.), Textbook of Small Animal Surgery (3a ed.). Saunders Company, 1737-1741 p.

Kuwahara, J. (1986). Canine and Feline aural hematoma: clinical, experimental, and clinicopathologic observations. American Journal Of Veterinary Research, 47(10): 2300-2308.

Lanz, I. O., \& Wood, C. B. (2004). Surgery of the ear and pinna. the veterinary clinics of small animal practice, $34(2): 567-599$.

Mccarthy, P. E. \& Mccarthy, R. J. (1994). Surgery of the ear. Veterinary Clinics Of North America: Small Animal Practice, 24(5): 953-969.

Mikawa, K. et al. (2005). Epidemiological and Etiological Studies on 59 Aural Hematomas of 49 dogs. Japanese Journal Of Veterinary Anesthesia and Surgery, 36(4): 87-91.

Pachaly, J. R. et al. (2021). Corticoterapia intralesional no tratamento de otohematoma em cães. Acta Scientiae Veterinariae, 49 : 1785.

Rodrigues, N. M. et al. (2019). Casuística cirúrgica em cães em um Hospital Veterinário - Universidade Federal do Piauí. Revista Brasileira de Pesquisa Veterinária e Zootecnia, 56(1): e146660.

Santos, F. F. \& Guimarães, J. P. (2020). Estudo retrospectivo das otites em cães e gatos atendidos no hospital veterinário em Santo/SP. ARS VETERINARIA, 36(3): 195-200. http://dx.doi.org/10.15361/2175-0106.2020v36n3p195-200.

Santos, S. I. R. (2008). 78f. Otohematoma canino: epidemiologia e terapêutica. Dissertação de mestrado em medicina veterinária, universidade técnica de lisboa. 
Research, Society and Development, v. 10, n. 8, e56310817338, 2021

(CC BY 4.0) | ISSN 2525-3409 | DOI: http://dx.doi.org/10.33448/rsd-v10i8.17338

Teixeira, C. R. et al. (2002). Estudo Epidemiológico do Otohematoma Canino. Revista Brasileira de Ciência Veterinária, 9: 172-174.

Valle, A. C. V., Kanayama, C. Y. \& Nader, T. T. (2015). Tratamento homeopático de otohematoma em cão: relato de caso. Revista Unimar Ciências, 24(1-2).

Yoshida N., Naito F. \& Fukata, T. (2002). Studies of certain factors affecting the microenvironment and microflora of the external ear of the dog in health and disease. Journal of veterinary medicine science. 64(12):1145-147. 